\title{
Chapitre 12 La caractérisation différentielle du Discours par ses autres : images et reflets configurants de la RDA
}

\section{Introduction Positivité du négatif : frontières « instituantes »}

"L'être se pose en s’opposant » : indéfiniment reprise ou reformulée, la formule de Hegel n'en a pas pour autant perdu de son pouvoir éclairant...

C'est - hors de toute visée de présentation de cette pensée et de celles qu'elle a inspirées - à la « vulgate » post-hégelienne, d'une positivité de la négation que je fais référence ici ; envisagée en termes de limite, frontière, séparation, différence, distinction, même/autre... comme productrice d'identité, elle s'énonce aisément sous forme aphoristique : «l'identité s’atteint par la différence ; autrui est la condition essentielle de la conscience de soi ; la négation est nécessaire au devenir soi..."

Elle s'impose - autant à la réflexion philosophique que dans le champ des sciences humaines ${ }^{1}$ - comme " envers » de l'indifférenciation, de la (con)fusion, de l'incorporation... : panorama qu'il serait vain de tenter d'évoquer par un florilège de « vraies » citations (par rapport à l'anonymat des aphorismes ci-dessus), tant le caractère " instituant » du négatif qui, formulé à ce niveau de généralité, peut être considéré comme partagé, s'est, en effet, en fonction des cadres de pensées, des problématiques, des objets divers, chargé de significations et d'enjeux si dissemblables - Bakhtine, Heidegger, Sartre, Ricœur, Bourdieu... - que le rapprochement de formulations d'apparence voisine, non contextualisées serait trompeur.

Deux citations ${ }^{2}$ seulement ici, l'une parce qu'elle émane de Benveniste et qu'il s'agit de langage... :

1 Outre la « distinction » travaillée par Bourdieu (« Exister, symboliquement, c’est différer » rappelle-t-il dans un entretien avec C. Levi-Strauss de 1988 (You tube), citons, par exemple dans le champ des sciences humaines F. Hartog, Le Miroir d'Hérodote-Essai sur la représentation de l'autre Gallimard, 1980, liant crucialement la question de l'altérité à celle de la frontière (p. 135, par exemple), ou E. Terray dans Penser à Droite (Galilée, 2012) distinguant l'investissement, xénophobe « identitaire », de ce qui est limite nécessaire à une identité (p. 110 sq., notamment) ou encore L'éloge des frontières de R. Debray (2010).

2 Dont j'emprunte la « rencontre » à S. Bikialo (2012: 122).

2 Open Access. (C) 2020 Jacqueline Authier-Revuz, published by De Gruyter. (cc)BY-NC-ND This work is licensed under the Creative Commons Attribution-NonCommercial-NoDerivatives 4.0 License. https://doi.org/10.1515/9783110641226-021 
La conscience de soi n'est possible que si elle s'éprouve par contraste [1966 : 260],

l'autre du philosophe Clément Rosset :

Il ne saurait donc être de moi que de l'autre et par l'autre, dont l'étayage assure l'éclosion et la survie du moi [1999: 48],

parce que à travers ses mots " étayage, assurer, éclosion, survie » apparaît nettement que la constitution du moi par différence d'avec l'autre n'est pas une « donnée » de fait, statique, mais, dans un « jeu de forces », un équilibre à produire et, toujours mouvant, à maintenir.

Délimitant, dans l'un du Discours-en-train-de-se-faire, le territoire de ses autres, reconnus pour tels, la RDA est apparue comme un geste méta-énonciatif dissimilateur, traceur de frontières. Les « émotions » observables chez les énonciateurs lorsque ce tracé séparateur se fait problématique, incitent, on l'a dit, à en questionner l'économie - discursive et énonciative - au-delà de celle, première, de doter tout dire de la possibilité, métalangagière, de parler d'un autre dire, autant que de tout autre référent.

Dans l'opération globale de "présentation de soi » inhérente au discours, la RDA apparaît, par la double spécificité de son statut métalangagier et de sa négativité (différentielle), comme un ressort crucial du « positionnement » des discours (1). Sa fonction configurante du Discours « par ses autres » s'accomplit indirectement par le jeu d'images et de reflets que des visées discursives diverses disposent en lui dans sa traversée du déjà-dit (2).

La RDA, partie prenante des mécanismes de négativité instituante (1), apparaît comme porteuse d'une figuration différentielle assurant l'identité du discours et du sujet qui l'énonce.

Ce mécanisme s'exerce sur deux plans du dire, distincts et solidaires, qu'on envisagera séparément, en tentant de saisir leur articulation au sein de la fonction configurative de la RDA : celui, au plan discursif, de la caractérisation différentielle du discours par les autres discours qu'il représente en lui, qui fait l'objet de ce chapitre et, en deçà, celui, au plan du rapport du sujet au langage, abordé ci-dessous 2.3.4 et envisagé aux chapitres suivants, d'une délimitation qui institue, dans la présence constitutive de l'ailleurs discursif, une parole "propre». 


\section{Mécanismes d'identification discursive et RDA}

\subsection{Ethos, identité discursive, présentation de soi.}

\subsubsection{La dimension langagière de la figuration de soi.}

Le champ est immense - de la rhétorique d'Aristote aux travaux contemporains de la prise en compte de cette composante obligée de toute parole qu'est l'« image », la «figuration », la « mise en scène » de soi qui, productrice d'une « identité discursive » est partie prenante de son effectuation. Je ne tenterai pas de le parcourir, renvoyant, par exemple, au panorama qu'en propose R. Amossy (2010), sous le titre «La présentation de soi - Ethos et identité verbale » et, dans le Dictionnaire d'Analyse de Discours, de Charaudeau et Maingueneau (2002), au jeu croisé des articles qui y introduisent : « ethos, face, identité, individuation, places (rapport, système de), portrait discursif, positionnement, rhétorique, rôles, aire d'énonciation, stratégie de discours... ".

Les pensées de la production d'images de soi comme composante de la vie sociale à travers toutes ses formes - vêtements, habitation, " manières de table », ancrages et trajectoires professionnels, régionaux, " appartenances » diverses, modes plus ou moins ritualisés de la sociabilité, etc., parmi lesquelles les pratiques langagières - comme celles de « la mise en scène de la vie quotidienne » de Goffman (1973) ou de la « distinction » de Bourdieu (1979) - ont évidemment nourri, à des degrés divers, les problématiques évoquées ci-dessus. Mais, celles-ci, en deçà des différences certaines qui les traversent, en termes, par exemple, des « places » - effectives et imaginaires occupées par les interlocuteurs, évoquées par Pêcheux et Fuchs (1975) ${ }^{3}$ et retravaillées par Flahaut (1978) des « images » de celui qui parle et de celui à qui le discours s'adresse, telles que les propose le « dispositif d'énonciation » de E. Véron (1984 : 35), d'identité socio-discursive chez Charaudeau (2009), de l'ethos produit aux divers plans de la « scène d'énonciation » chez Maingueneau (1993, 2012), partagent, à l'instar de la rhétorique d'Aristote, une même focalisation sur la dimension langagière de la figuration de soi, dont R. Amossy (2010 : 210) souligne les « deux aspects cruciaux » : « la construction d'une identité verbale et la quête d'une efficacité rhétorique ».

Ainsi retrouve-t-on la panoplie des marqueurs identitaires, évoqués par Goffman, autant comme " ressources » que comme "stigmates » dans le jeu social, sous les espèces des indicateurs langagiers d'appartenance sociale, régio-

3 Présenté in Maingueneau (1976 : 143) sous le titre « Places et “formations imaginaires” ». 
nale, générationnelle, " genrée », culturelle, politique, idéologique, professionnelle... et " générique » - au sens de genre de discours - du sujet énonciateur, et du discours qu'il tient, analysés par un riche ensemble de travaux ${ }^{4}$.

Parmi les éléments langagiers ${ }^{5}$ retenus par les analyses d'un discours en tant que lui conférant une identité, on peut noter (sans que soit tout à fait négligés les aspects de variété lexicale ou syntaxique, les traits de prononciation comme les élisions familières par exemple) que l'ensemble de ce qui est classiquement reconnu comme «formes d'expression de la subjectivité » se taille la part du lion : présence/absence du je, des formes renvoyant à l'allocutaire $t u$, vous et termes d'adresse, du on, des modalités interrogatives, exclamatives, de l'effacement énonciatif...

La focalisation sur ces éléments, certes saillants, ne doit pas faire oublier que c'est tout qui, dans un discours, concourt à son individuation - ce que souligne la remarque de J.-M. Barberis ${ }^{6}$, estimant que par la focalisation sur les « marques de l'énonciation » :

on se prive de la possibilité de relier le sujet égotique, pleinement individualisé, aux autres formes d'apparition de la subjectivité, beaucoup plus discrètes et diffuses [les formes les plus saillantes devenant] les arbres cachant la forêt où se trouvent tous les autres modes de positionnement linguistique de la subjectivité.

\subsubsection{Caractères de l'identité produite par le discours}

Avant (1.2) de préciser en quoi la RDA se spécifie dans l'ensemble des faits envisagés comme porteurs d'identité discursive, on peut rappeler quelques propriétés reconnues, en deçà de ce qui distingue les diverses approches, à cette identité produite par le discours, et que la RDA partage :

$4 C f$., par exemple, dans la très vaste bibliographie relevant de cette problématique, plusieurs recueils qui en explicitent nettement la visée : La production d'identité (Colloque de Sommières 1986), Images de soi dans le discours (Amossy dir. 1999), Identités sociales et identités discursives du sujet parlant (Charaudeau dir. 2009).

5 Sans que, entre les éléments non langagiers (tenue vestimentaire, apparence physique...) accompagnant le discours et les éléments strictement linguistiques, l'espace du mimo-gestuel, de la voix, du débit soit partageable de façon discrète.

6 (Détrie et al., 2001 : 330) ; ainsi que sa reprise, en termes d'ethos par M. Suchet (2014 : 183), notant, dans sa riche étude des « figures d'énonciation hétérolingues » qu'elles sont susceptibles d'apparaître à " n'importe quel point de la chaîne du discours, même en l'absence des indices habituels de la présence d'un "sujet” ». 
- l'identité, l'image de soi qui accompagne le discours, n'est pas une « essence », un donné, mais relève de la dynamique d'un processus de « construction » ou « production langagière d'identité ${ }^{7}$;

- cette identité, produite dans et par le langage, est étrangère à la permanence, la stabilité, la fixité... ; jamais " acquise », elle est à maintenir ${ }^{8}$, elle est à refaire, rejouée dans chaque parole : mobile, fluctuante dans le cours d'un même discours, elle est aussi plurielle, pour un sujet donné porteur d'identités multiples au gré des places diverses qui lui sont offertes ou imposées par le discours ${ }^{9}$;

- le processus de production d'une identité énonciative s'accomplit à travers tous les degrés de conscience du sujet énonciateur : de certains marquages identitaires - accents, variété de langue - échappant tendanciellement au contrôle du sujet parlant ${ }^{10}$ aux stratégies concertées « d'individuation volontaire » dans le champ politique notamment ${ }^{11}$;

- cette identité produite est celle d'un positionnement discursif ${ }^{12}$, un placement qui s'opère contrastivement par rapport au milieu discursif environnant ; les « indicateurs » identitaires - déictiques, modalités, syntaxe, lexique, etc. - que présente un discours le font reconnaître, sur fond de discursivité ambiante,

7 Cf. par exemple Lafont (1986: 8) : «L'identité n'est pas plus un donné qu'une essence : c'est le résultat d'une production signifiante que la société langagière permet, règle, contrôle »; ou Amossy (2010 : 210) : «L'identité n'est pas une essence qui se traduit sur un mode plus ou moins authentique et qu'on peut exhiber ou au contraire dissimuler pour des besoins stratégiques [...], mais une construction verbale effectuée dans l'échange ».

$8 \mathrm{Cf}$. Maingueneau évoquant «l'instauration et [le] maintien d'une identité énonciative » (DAD $2002: 453)$.

$9 \mathrm{Cf}$. le « potentiel de fragmentation identitaire » évoqué par N. Labrie « Nous disposons tous, en effet, d'identités multiples qui peuvent justifier notre affiliation à diverses communautés » ; ou la " démultiplication constituante de chaque être parlant, de chaque sujet, qui le diffracte et le pluralise en autant d'échos décalés, de discours et de langage différenciés » évoquée par J.-M. Prieur (1986 : 303) en écho à Freud : « Chaque individu fait partie de plusieurs foules, présente les identifications les plus variées [...] participant de plusieurs âmes collectives, de celles de sa race, de sa classe, de sa communauté confessionnelle, de son état, etc. » [Essais de psychanalyse, Payot, p. 157]. $10 C f$. par exemple le " marquage identitaire peu conscient et très fortement ressenti » d'un accent régional, signalé par Guespin (Colloque de Sommières, 1986 : 253).

11 Mises au jour, par exemple, dans les discours à visée auto-identificatrice et « différenciatrice » du Congrès de Tours, par Marcellesi et Gardin (1974 : 214, 234-236).

12 Caractérisé comme « catégorie de base de l'AD » par D. Maingueneau (DAD 2002 : 453); le " positionnement » est envisagé comme recouvrant aussi bien un placement dans un champ structuré conflictuellement - politique, idéologique, scientifique... - qu'une position occupée, plus ou moins consciemment, dans un espace de valeurs et de normes comportementales, par les sujets sociaux. 
tant au plan générique, comme programme électoral, mode d'emploi d'un appareil, lettre familiale, article scientifique ou discussion de comptoir... qu'au plan individuel où l'énonciateur se « positionne » différentiellement comme expert, mère de famille, chrétien de gauche, jeune « branché », « macho »...

\subsubsection{La RDA « indicateur d'identité », parmi d'autres}

La RDA que présente un discours - degré de présence, autres convoqués, formes de la représentation... - fait partie, au même titre que tous les autres aspects d'un discours, des éléments par lesquels celui-ci se positionne par rapport à d'autres discours dans le champ discursif.

Ainsi, par exemple, l'absence (a) ou, au contraire, la profusion (b) de faits de RDA dans un discours peut-elle être corrélée avec une énonciation « sans énonciateur » (a) ou, au contraire, à « je-moi » envahissant (b), dans le positionnement énonciativo-discursif opposé de modes d'emploi ou textes de loi (a), et d'interactions orales à fort enjeu narcissique comme les échanges entre pairs (b) analysés par Goodwin (1989) ou Vincent et al. (1997), ou les récits dont J. Bres (1994 ; cité chap. 5 (28)) souligne la dimension de " production d'identité sociale ».

La pertinence de la prise en compte des faits de « discours rapporté » parmi tous les autres « indicateurs » d'identité génériques, idéologiques, individuels dans les approches de discours menées explicitement en termes de présentation de soi ou de positionnement est ainsi rappelée par D. Maingueneau ${ }^{13}$ :

On ne cite pas de la même manière dans une revue de physique nucléaire et dans une conversation, dans un quotidien dont le public cible est une élite et dans un quotidien populaire.

qui souligne que

la manière dont une parole est attribuée à une autre source est solidaire des caractéristiques de l'ensemble du discours citant.

et, identifiant la RDA comme « une des dimensions du positionnement » (idt) ${ }^{14} \mathrm{du}$ discours, la pose comme « modalité à part entière du fonctionnement discursif » (idt $)^{15}$.

13 Article « Discours rapporté » (DAD 2002 :194-195).

14 Ce que reprend l'article « Positionnement » du même ouvrage où, à côté du « genre », c'est la « manière de citer » qui est retenue parmi les diverses dimensions du discours susceptibles de «manifester » son positionnement.

15 Maingueneau (1981 : 140), position pertinemment illustrée par l'analyse contrastive des discours religieux du grand siècle, « humaniste dévot » et janséniste, menée in Maingueneau (1983). 
Le lien entre « discours rapporté » et « production d'identité » est également thématisé par R. Amossy (2010) consacrant dans son parcours des « modalités verbales de la présentation de soi » une section (p. 148 sq.) aux « jeux de l'ethos dans le discours rapporté en situation interactionnelle ».

Dans le riche ensemble de travaux illustrant cette perspective, je me contenterai d'évoquer, à titre d'exemple, quelques-uns de ceux qui, dès leur titre, affichent la problématique «RDA et image de soi »- tels « Discours rapporté, représentations sociales et présentation de soi » de D. Vincent (2004), « La Parole de l'autre comme construction identitaire » de A. Aït-Salia Benaïssa (2004) ou « La polyphonie au service de l'ethos » de H. Constantin de Chanay (2010).

\subsection{Le positionnement discursif par la RDA : métalangagier et différentiel}

Le discours rapporté est, on l'a vu, largement reconnu comme participant, dans le discours, à la production d'un ethos, d'une présentation de soi, parmi et au même titre que nombre d'autres faits langagiers - déictiques, modalités, lexique... Il convient, à présent, de dégager ce qui dans l'ensemble des marqueurs d'identité langagière d'un discours, fait la spécificité - « configurative » - de la RDA. Cette spécificité tient aux caractéristiques de la RDA : son statut métalangagier qui lui permet la représentation, interne au Discours de $\mathbf{L}$, d'une altérité discursive.

\subsubsection{Du dire sur le dire - un fait de métadiscours}

On a vu que c'est à travers ce qu'il montre - son lexique, ses stéréotypes, son accent, ses connecteurs argumentatifs, etc. - que le discours se positionne, non par ce que l'énonciateur dit de lui-même et de son discours : c'est la dimension de l'ethos de la présentation de soi, dont D. Maingueneau (2002 : 3) et R. Amossy (2010 : 35, 113-117) soulignent la pérennité, depuis Aristote qui « entendait par là l'image que donnait implicitement de lui un orateur à travers sa manière de parler ${ }^{16}$, jusqu'aux approches contemporaines de Barthes (1966:212) ou Ducrot (1984 : 201), notamment, que D. Maingueneau reformule ainsi

L'efficacité de l'ethos tient au fait qu'il enveloppe en quelque sorte l'énonciation sans être explicité dans l'énoncé. [...]. [...] l'ethos se montre dans l'acte d'énonciation, il ne se dit pas dans l'énoncé. Il reste par nature au second plan de l'énonciation : il doit être perçu, mais ne pas faire l'objet du discours. [Maingueneau, 2002 : 57].

16 Maingueneau (1996: 39). 
Dans l'ensemble des éléments susceptibles de jouer dans la production de l'image d'un discours et de son énonciateur, le plan langagier dispose, seul, de la capacité réflexive de se prendre pour objet : on peut faire, au plan vestimentaire, de l'« exhibition identitaire », comme, au plan langagier, on peut " afficher » telle variété de langue ; mais seul le discours permet de passer de la simple présentation/monstration au plan second - langage " au carré », si l'on veut - d'une représentation de discours. Il en va ainsi sur les deux versants du métadiscours que sont, dans le Discours, son auto-représentation (ARD) et la représentation d'un discours autre (RDA).

On peut mettre en regard un énoncé (a) et ses deux avatars, métadiscursivement accompagnés d'ARD (a') et de RDA (a") :

(a) Cette décision est une grave erreur.

(a') Cette décision, je le dis en conscience après mûre réflexion, est une grave erreur.

(a") Cette décision est, n'en déplaise à $\mathrm{M}$. X qui la « salue comme un progrès décisif », une grave erreur.

Ces formes parlent du dire - Discours en train de se faire en (a'), autre discours mis en rapport avec le premier en (a"). Il n'est pas question pour autant de dire - allant contre le fait que l'ethos se montre mais ne se dit pas dans l'énoncé - que ces formes « disent l'ethos ». Elles sont bien partie prenante - au même titre que son niveau de langue par exemple - de la «manière de parler » que le Discours présente et à travers laquelle se produit son image. Mais on est amené à questionner la spécificité du rôle que, dans le processus de production d'image ou de « mise en scène » qui s'opère dans un discours via les formes qu'il " présente ", jouent celles que le discours présente en tant qu'elles le représentent, donnant, réflexivement, sur un mode susceptible de se charger de théâtralité, des images explicites - directes (ARD), ou indirectes (RDA) - à travers celles des discours qu'il reconnaît comme « ses » autres.

\subsubsection{L'ARD : limites de la caractérisation directe du dire}

Visant à préciser le fonctionnement propre à la RDA dans la production métadiscursive d'une image du Discours et de son énonciateur, je n'envisagerai, et sommairement, de l'autre versant - celui de l'auto-représentation du Discours (ARD) - que quelques points sur lesquels il s'oppose au premier, permettant d'amorcer la caractérisation de la RDA.

A priori, la caractérisation ${ }^{17}$ explicite, directe, positive, par laquelle les formes de l'ARD redoublent de son image, comme « en miroir », le dire en train de se faire,

17 Rappelons qu'il ne s'agit pas ici de caractérisation explicite de la personne de l'énonciateur, telle le « je suis très modeste », objet de plaisanterie ( $c f$. Amossy, $2010: 115$ ). 
pourrait apparaître comme instrument par excellence de la production discursive d'identité.

On a esquissé, (chap. 1) la variété des formes par lesquelles un dire peut, sur une " portée seconde ", revenir en boucle sur lui-même ${ }^{18}$ : formes phrastiques (1) ou circonstancielles (2), portant sur le contenu du dire (a) ou une manière de dire (b), comme :

(1a) Je te dis que $P$

(1b) Je dis ce mot avec des guillemets.

(2a) S'il faut tout dire

(2b) Pour ainsi dire

Je n'entends pas, ayant consacré une étude détaillée ${ }^{19}$ à la variété des inscriptions modalisantes du dire, au plan de ses manières de dire (du type (1b, 2b), dans les jeux de non-coïncidence de l'interlocution, de la référenciation ou de la plurivocité20 ${ }^{20}$ tant dans l'inventaire des formes qu'à ce que leur usage révèle de l'économie énonciativo-discursive des discours, minimiser l'intérêt de «l'entrée » dans les discours par le mode selon lequel ils occupent (volontiers, rarement, jamais..., pour quel type de commentaire...) cette position méta-énonciative de surplomb sur le dire propre ${ }^{21}$. Mais, paradoxalement, le pouvoir de production discursive d'une image de soi apparaît, sur ce versant ARD du métadiscours, comme restreint par la stricte centration réflexive $d u$ « je dis » sur le dire en train de se faire. Ainsi peut-on noter que :

- le caractère non nécessaire de ce qui relève du redoublement, en miroir, du dire par lui-même, induit, aisément, le soupçon d'une tendance à l'emphase, à l'affectation, ou un penchant à la complaisance - imputable à qui « s'écoute parler » comme on dit...- freinant le recours à l'ARD ;

- le spectre des caractérisations de son propre dire, admissibles au regard de normes de bienséance communicationnelle ou de cohérence énonciative, est restreint relativement à celui que parcourt la caractérisation d'un dire quelconque autre, que ce soit, par exemple, dans l'ordre de la louange :

18 Cf. 1.2.3.1 (iii), p. 22, formes, rappelons le, n'incluant pas les performatifs par lesquels l'acte de dire ne se réalise pas « redoublé » de son explicitation, mais via cette explicitation.

19 Authier-Revuz (1995/2012); cf. ci dessus chap. 8.3.1 et 8.3.2, p. 291.

20 Comme, respectivement, dans « si tu vois ce que je veux dire », « c'est le mot qui convient », « aux deux sens du mot », par exemple, qui, contrairement à la MAE n'impliquent pas la mise en jeu d'un « discours autre ».

21 Non plus que l'intérêt du vaste champ des formes méta-énonciatives, "non opacifiantes », portant (comme 2a) sur le fait ou le contenu du dire. 
(3) RDA Ce qu'il appelle, admirablement, « $\mathrm{X} »$.

ARD ? Ce que je vais appeler, admirablement, « $\mathrm{X} »$.

comme de la critique :

(4) RDA Il a eu l'indécence/l'obscénité de dire...

ARD ? J'ai l'indécence/l'obscénité de dire...22

- enfin, si la grammaticalité et l'acceptabilité de formes d'ARD - telles les « boucles » de modalisation autonymique largement amplifiées ou joueusement récursives par exemple ${ }^{23}$, ou les interminables successions de circonstants métadiscursifs d'un je dis retardant l'arrivée du dire du $\mathrm{P}$ annoncé ${ }^{24}$ manifestent la liberté créative offerte à leur énonciateur, il n'en reste pas moins que l'ARD relève d'une pratique assez fortement stéréotypée par le nombre et la fréquence d'emploi d'expressions figées, telles, notamment, les associations :

je dis + modaux devoir, pouvoir, vouloir...

ou les formules comme :

si vous voulez, passez-moi l'expression, pour ainsi dire, c'est le cas de le dire,...

\subsubsection{Ressorts et ressources propres de l'opération métadiscursive de figuration du Discours par « de l'autre "}

Une figuration explicite, différentielle, interne au Discours

Le « positionnement » qui s'opère en tout discours est, rappelons-le, selon la présentation qu'en propose D. Maingueneau ${ }^{25}$ :

22 Acceptable à nouveau, dès lors que par un effet de reprise contextuelle immédiate, ou un marquage intonatif ou typographique, la caractérisation de ma parole comme indécente serait entendue comme celle d'un autre - dans une MAE non (allusion) ou peu marquée.

23 Voir le parcours des boucles revenant sur " ces mots qui ne vont pas de soi » (Authier-Revuz, 1995/2012). Je n'en reprendrai ici que - doublement « bouclante » - l'une de celles citées en exergue : «Ah, non, changer des bébés toute la journée, moi je trouve ça emmerdant,... au sens propre d'ailleurs, enfin, propre [rires] si on peut dire » [Conversation train (jeunes filles parlant du métier de puéricultrice), oct. 1984].

24 Telle : "Je dis, à regret mais en conscience, en pesant mes mots, ne renonçant pas à faire entendre la voix du bon sens et en espérant qu'elle soit reconnue par ceux qui ont conservé la liberté de leur jugement, etc. $\mathrm{P}$ ».

25 DAD 2002: 453. 
Le fait qu'à travers l'emploi de tel mot, de tel vocabulaire, de tel registre de langue, de telle tournure [...] un locuteur indique comment il se situe dans un espace conflictuel.

assortie d'exemples :

en utilisant la lexie « lutte des classes » on se positionne comme de gauche - en parlant d'un ton didactique et avec un vocabulaire technique on se positionne comme spécialiste, etc.

Si on met en regard, en termes de positionnement du discours, un usage "simple » de la lexie « lutte des classes », du type (5) et un emploi en ARD comme (6) avec des emplois « en RDA » comme (7) ou (8) :

(6) ARD Je dirais que cette affaire est, une fois de plus, un épisode de la, utilisons le mot propre, lutte des classes.

(7) RDA C'est une question d'équité, de morale, pas de « lutte des classes », comme disent les nostalgiques du marxisme [oral, juin 1995].

(8) RDA Pour lui, quelle que soit la question, la Syrie, Trump, la dette des états, le réchauffement climatique, je t’en passe, il a une seule réponse, "la lutte des classes », sans doute à un certain niveau il a pas tort, mais à ce degré de généralité, ça t’avance pas beaucoup... [oral, 10-12-2016].

il apparaît que la spécificité du mode sur lequel la RDA s'inscrit dans la " production par le discours d'une image de soi » tient à la conjonction de trois caractères. Cette figuration que la RDA opère dans le discours est en effet :

- explicite : contrairement aux « indicateurs » d'identité relevant d'une individuation «montrée » (cf. (5)), elle passe (comme l'autre mode métadiscursif, l'ARD, par du dit ;

- différentielle : le méta-dire de l'ARD accompagne le fait que - et la façon dont - un dire est en train de se faire d'une représentation directe et positive de celui-ci (cf. (6)) ; avec la RDA - puisque ce sont des éléments de discours autres, « dissimilés » comme tels au sein du Discours, qui sont représentés la production d'une image de soi par le discours emprunte un parcours indirect, à travers lequel l'image du Discours se dessine négativement, par différence ;

- interne au discours : la dimension contrastive est, à l'évidence, au principe même des processus de présentation de soi, ou de positionnement ; mais ( $c f$. (5) par exemple) c'est sur fond de discursivité environnante, dans l'« espace conflictuel » où il se produit qu'un discours, relativement à des discours extérieurs, s'identifie, différentiellement, à travers les éléments langagiers qu'il «présente ». Avec la RDA, le Discours convoque le « comparant » extérieur à l'intérieur, met en place - et en scène - les autres qu'il choisit de représenter 
en lui : ainsi, le Discours trouve-t-il en chaque forme de RDA, qui articule, sur la chaîne, de l'un et de l'autre, un lieu où, par le rapport qui s'y établit à un discours différent, il peut marquer un trait de son positionnement, de son image.

Remarque 1 : L'altérité de l'interlocuteur : présente ou représentée ? Relativement au processus de positionnement-individuation propre à la RDA - (1) de représentation, (2) d'une altérité, (3) interne - duquel se différencient les « indicateurs » d'identité (fonctionnant relativement à des autres extérieurs) et l'ARD (représentation interne mais non différentielle), le statut de l'autre-interlocuteur est complexe.

Le rapport interlocutif fait jouer, dans le dialogue, une altérité interne à celui-ci, mais, si saillante qu'elle puisse apparaître (polémique, registres de langue fortement différenciés...), comme dans :

(9) A : Cette mesure est indispensable.

B : C'est une belle connerie, oui !

elle n'est pas assortie d'une représentation, c'est-à-dire de la production, en discours, d'une image d'altérité par rapport à laquelle se constitue une image de soi. La différence entre les deux discours se « présente » par leur rencontre (ici articulée par l'anaphore).

Mais le discours de l'interlocuteur peut aussi - qu'il vienne d'être tenu dans l'échange, ou énoncé loin avant ou en dehors de celui-ci, ou évoqué comme virtuel... - être pris pour objet de RDA de la part d'un partenaire de l'échange. Les effets emphatiques de différenciation d'avec l'interlocuteur que permettent la mise en scène de l'échange ont été analysés, par exemple, dans un débat de candidats à l'élection présidentielle ${ }^{26}$ : les auteurs notent que c'est lorsque l'un des interlocuteurs entend « se démarquer très nettement du discours de l'autre » qu'il passe (au-delà de la simple expression d'un désaccord comme ci-dessus (9), à la représentation du discours qu'il réfute - ici de surcroît objectivé en il dit, de préférence à vous dîtes :

Jospin : [...] Lui pense, et il l'a dit, qu'au fond il y a une sorte de dérive [...]. Moi, j’ai un point de vue différent : je pense que [...]. [...] il dit : Il suffit que l'homme qui sera président de la République [...]. Moi, ma vision est très différente [...] (p. 116, soulignements des auteurs).

Cette double possibilité, très sommairement évoquée ici, pour le discours de cet autre particulier qu'est l'interlocuteur, d'apparaître comme représenté ou, simplement, comme présent, factuellement, le distingue du discours autre du « tiers parlant » qui, désigné aussi comme « tiers absent », ne peut apparaître que s'il est représenté. En regroupant au titre de " discours autres, qu'ils soient tenus par l'interlocuteur ou par un tiers absent $»^{27}$, ces deux types d'altérité, il faut prêter attention au fait que ce n'est pas de la même façon qu'opère, dans les deux cas, le processus d'individuation « par lequel tout sujet parlant cherche à se construire une identité », selon qu'il passe, nécessairement ou non, par la représentation de l'autre.

26 Débat Chirac-Jospin en 1995, étudié in Sullet-Nylander et al. (2011 : 115-119).

27 Charaudeau (DAD 2002 : 307-308). 


\section{Variété illimitée des contacts entre le Discours et ses discours autres.}

La comparaison avec l'ARD - et la représentation directe et positive qu'elle effectue - souligne les ressources propres à la caractérisation différentielle et relationnelle d'un Discours par sa RDA. Le spectre des réalisations de la RDA est en effet illimité : au référent unique et imposé de l'ARD répond l'infinie diversité des référents représentés comme " dire autre »- actuels/virtuels, passés/présents/à venir, singuliers/pluriels/collectifs, oraux/écrits, etc. ( $c f$. chap. 1), déployant la variété non dénombrable des " parlers " volontiers parcourue par Bakhtine et figurant dans des situations irréductiblement singulières. Et, si l'auto-représentation, soumise à des restrictions d'acceptabilité, apparaît comme relativement contrainte, la représentation, dès lors qu'il s'agit de discours autre, ne rencontre, en soi, aucune limitation ni censure ${ }^{28}$.

Ainsi, la variété des rapports susceptibles d'être mis en scène entre les discours autres et le Discours qui les convoque est-elle, comme celle de ces discours, potentiellement infinie. La variété illimitée des modes sur lesquels s'exerce dans les Discours cette capacité à " avancer » métadiscursivement accompagnés d'autres discours de leur choix, fait de la RDA un ressort crucial - apte aux nuances les plus fines dans ses opérations de mise en rapport différentielle de l'un et de ses autres - du positionnement énonciativo-discursif des Discours ${ }^{29}$.

\section{La RDA : visées discursives et fonction identifiante}

La RDA, observable dans un discours, suscite, classiques toutes deux et pertinentes, deux réactions : celle d'une question "Pour quoi faire? ", dans quelle intention, comme élément de quelle stratégie fait-on, dans un discours, adressé à un autre, appel à un «tiers parlant $»^{30}$ ? et celle : «Dis-moi qui tu cites, je te dirai qui tu es ", d'un projet, ou promesse, de déchiffrer, révéler, derrière ce qui s'ex-

28 Sinon, bien entendu, à un autre niveau, celle des normes requises par un genre, une situation - pour la RDA comme n'importe lequel des aspects du discours.

29 Prendre la mesure du pouvoir singularisant, pour un Discours, qu'offre l'extraordinaire diversité de la RDA qu'il présente passe par une investigation systématique du réseau des paramètres dans lequel elle s'inscrit : place faite au discours autres ? Quels autres ? Combien ? Saisis dans quel rapport ? Représentés par quel mode ?... à laquelle cet ouvrage ne consacre qu'une esquisse programmatique ( $c f$. chap. 14 et 15)

30 Question mise en évidence dans le titre « Citer pour quoi faire ? Pragmatique de la citation» du recueil de Jaubert et al. (2011) - dans lequel « citer », comme dans « Dis-moi qui tu cites... » et diverses formulations reprises dans ce chapitre, a bien entendu le sens «étendu » (III) évoqué dans l'appendice au chap. 9, emploi auquel pour cette raison je me conformerai dans ce chapitre. 
pose - ces autres discours représentés -, une vérité plus discrète, voire secrète, y compris peut-être pour l'énonciateur L, que pourtant elle concerne. Là où la première s'interroge sur la cause d'un recours à la RDA - ce qui dans l'avancée de son discours le motive pour son énonciateur $\mathbf{L}$-, la seconde, envisageant ce que produit cet appel à de «l'autre discours », en pointe un effet, non nécessairement calculé.

\subsection{Le « Faire » de la RDA et son « ce faisant »}

C’est toute une panoplie de stratégies rhétoriques, pragmatiques, communicationnelles, qu'a fait apparaître, dans de nombreux travaux, l'observation de la mise en œuvre de la RDA dans la diversité des discours.

Sans viser nullement à un inventaire qui, de toute façon, ce niveau langagier ne relevant pas du système de la langue, ne saurait être fermé, pas plus que ne sont discrets ses éléments, on peut rappeler, classiquement reconnues et volontiers déployées dans leur diversité ${ }^{31}$, quelques unes des opérations qu'un Discours réalise en passant par un autre discours, relevant de :

- l'argumentation (débattre, adhérer, réfuter, élaborer un point de vue...), la persuasion (convaincre, emporter l'adhésion...), ces objectifs ayant recours à des processus de validation (conforter son dire par des appuis, garants, arguments d'autorité... $)^{32}$;

- la séduction (agrémenter un discours sur un mode ornemental - culturel, ludique...) ;

- la production et la transmission de connaissances et d'informations (dans le champ scientifique, médiatique...) ;

- la narration (de faits de discours réels ou fictifs) ;

- etc.

$31 \mathrm{Cf}$. par exemple, in Maingueneau (1991: $137 \mathrm{sq}$.), les fonctions - « relique, épigraphe, culture, preuve, autorité » - de la citation, in Quéré (1992 : 92-98) les variétés d'un « faire citationnel » relevant de l'argumentation, de l'alliance, de l'étalage, ou in Schneider (1985 : 279), les citations de pédanterie, d'aphasie, de culpabilité, de narcissisme...

32 Selon le mécanisme que L. Chetouani (2004) formule en " Faire dire pour dire », pointant - à travers le double jeu dans une déclaration à l'ONU de faits de " polyphonie consensuelle » et « conflictuelle » - « l'art politique de se servir de la voix d'Autrui pour faire passer ses propres idées », c'est-à-dire, au total, pour un énonciateur, d'emprunter le détour métadiscursif des $X a$ raison/a tort de dire $P$ pour dire $P /$ non $P$. 
La « présentation de soi » ou la « production d'identité » qui se réalise dans un discours via sa RDA n'est pas à ranger parmi les visées discursives, relevant de savoir-faire explicites ou exemplifiés dans les manuels de communication ou le recueils de discours " modèles », mais comme un processus qui s'accomplit, secondairement, à travers elles : un discours, un énonciateur représente des discours autres convoqués pour, en vue de "faire quelque chose » (valider, raconter...) et, ce faisant, il se présente lui-même, identifié par rapport à ces autres.

Ainsi est-ce pour raconter ${ }^{33}$ que le Cardinal de Retz ( $c f$. ci-dessus chap. 6 (45), p. 221) convoque incessamment, au fil des péripéties de la Fronde, les multiples dires - dont le sien - recueillis au cœur de l'action... et, ce faisant, il apparaît, très narcissiquement, comme figure centrale des évènements, tout à la fois acteur privilégié et observateur lucide.

C'est pour légitimer, crédibiliser, leur parole dans le cadre d'une argumentation parlementaire, en 1981, entre tenants de et opposants à l'abolition de la peine de mort, que les orateurs font appel de façon répétée à des discours autres ${ }^{34}$ et, ce faisant, qu'ils dessinent, en fonction des autres qu'ils se sont respectivement choisis à l'appui de leur position, des « images discursives de soi » : d'un côté, de porteur $\mathrm{du}$ " flambeau », transmis à travers l'histoire par des personnages illustres, Hugo, Jaurès, Camus... et, de l'autre, de porte-parole des « gens ordinaires », de la « France profonde », d'une « majorité silencieuse ».

On a évoqué (chap. 5.3.2, p. 161) les trois « cercles » de discours autre dont s'entoure - selon une disposition intertextuelle que présentent volontiers les écrits de « développement personnel » - un texte de psychologie appliquée à la « poursuite du bonheur » : dans sa visée foncière de persuasion, il enrôle, en nombre, des autres chargés de le valider - spécialistes, côté savoir ; patients, côté expérience clinique - et de le valoriser - grands auteurs porteurs d'aura culturelle - ; et, ce faisant, se trouve produite l'image, triplement positive, d'un discours énoncé par une personne compétente dans sa discipline, engagée dans l'écoute et le soin de la souffrance, et nourrie de sagesse humaniste.

Ce rapport d'un « faire » discursif, passant par la représentation d'autres discours, à un effet d'image de l'énonciateur et de son dire, s'observe comme préci-

33 Dans l'étude « Récit oral et production d'identité » que J. Bres (1996) consacre au vaste corpus d'entretiens avec des mineurs, racontant après coup, leur participation personnelle au long conflit social de 1980, on peut noter l'omniprésence de la RDA comme élément pertinent de cette production d'image de soi par le récit des interactions conflictuelles entre un « je » narrateur et des interlocuteurs représentants du patronat ou des forces de l'ordre notamment. $C f$. l'exemple (29) du chap. 5, p. 164.

34 Exemple que j'emprunte à l'analyse de Micheli (2006, 2007) déjà évoquée aux chap. 5.4.2, p. 191 et 11.3 (48), p. 462. 
sément normé au plan générique : il en va ainsi des textes de vulgarisation scientifique pour grand public ${ }^{35}$. La visée, génériquement affichée, de transmission de savoir, fait massivement appel à la représentation du discours de deux sortes d'autres : celui des « savants » (au DD, DI...), qu'il faut « faire passer », et celui $\mathrm{du}$ lectorat-cible, dans les mots « ordinaires » duquel (en MAE) il faut parfois, pédagogiquement et approximativement « traduire » le discours scientifique ${ }^{36}$ et, ce faisant se met en place dans cet « entre-deux autres » du Discours, l'image générique du vulgarisateur " parlant pour » les deux autres - à la place des uns, les savants, et en direction des autres, les lecteurs - comme " go-between », médiateur et « troisième homme » pour reprendre une des images fondatrices du discours des praticiens de la vulgarisation, se dotant volontiers de la « mission » (de « cohésion sociale ») de combler, en « rétablissant la communication », le « fossé » creusé entre la Science et les citoyens ordinaires.

Ainsi apparaît le caractère second, dérivé, du mode sur lequel s'opère la présentation de soi, le positionnement, de soi dans le Discours, à partir des discours autres convoqués par celui-ci, au service des objectifs qu'il poursuit ${ }^{37}$.

Plusieurs études consacrées à la fonction du "discours rapporté » dans le Discours débouchent, à travers des formulations diverses, sur cette dualité des plans où se joue l'action de la RDA : celui, immédiatement observable des stratégies discursives diverses qui la mettent en œuvre, et celui, reconnaissable, de la fonction, configurante, de production d'une image de soi, qui s'accomplit à travers le premier.

Ainsi H. Quéré (1992) évoquant divers rôles discursifs de l'appel à l'autre discours - « tirer argument du déjà dit [...], du déjà vu et approuvé », " marquer une dette », viser un « label de qualité », etc. - conclut-il : « Chacun donc, en citant, se situe » (Quéré 1992 : 94). M.-M. de Gaulmyn, marque aussi la différence, pour le Discours, entre ce qu'il dit et ce que « par là » il fait :

Donnant à entendre d'autres que soi, on se donne soi-même à voir. Les évaluations formulées sur les paroles d'autrui guident les appréciations que d'autres se forment sur nous (de Gaulmyn 1996 : 43)

35 Cf. notamment (dans une très vaste bibliographie) Mortureux (dir.) (1982) et de nombreux travaux traitant de la problématique de la « didacticité » (S. Moirand et la collection des Carnets du CEDISCOR)

36 Tels, empruntés à Authier-Revuz (1982b) : «[...] Il existe pour ces ions des sortes de "portes » minuscules - ou « canaux voltage-dépendants »-constituées [...]», «[...] des pili sortes de flagelles qui $[. .$.$] », « [...] entouré d'une « boite » protéique (la capside) [...] ».$

37 Ce caractère second de la production d'identité rejoignant le statut « indirect » et « différentiel » de la figuration de soi « par les autres » explicité ci-dessus 1.2.3. 
De même, M. Doury, s'attachant à « la fonction argumentative des échanges rapportés » aboutit-elle ${ }^{38}$, à travers l'analyse de ces échanges rapportés, à la mise au jour, derrière leur fonctionnement comme « support » de mouvements argumentatifs, du

rôle capital [qu'ils] jouent dans la construction de l'ethos - de l'image que le locuteur construit de lui-même à travers son discours. (Doury 2004 : 261)

Telle est aussi, centralement, la démarche de D. Vincent à propos du « recours au discours rapporté dans l'oral standard » : observant, notamment dans les récits de parole où - relevant d'une «stratégie fonctionnelle » - il « sert le plus souvent à des fins argumentatives ${ }^{39}$, l'auteur considère que les divers points de vue - narratif, argumentatif, interactionnel - selon lesquels on peut envisager les discours rapportés

sont autant de clefs pour accéder à ce que les individus veulent montrer d'eux-mêmes [et que] en réalité, peu importe la typologie [narrative, argumentative, etc.] que l'on adopte, presque toutes ces fins s'inscrivent dans une perspective de construction de soi. (Vincent 2004 : 244)

Enfin, au terme d'une préface récapitulant le riche parcours collectif de réponses apportées, en termes de « pragmatique de la citation », par des discours didactiques, scientifiques, politiques, médiatiques, à la question liminaire « Citer pour quoi faire ? », A. Jaubert et L. Rosier « mettent en avant»

la visée sub-pragmatique de la citation ${ }^{8}$ qui sous-tend en profondeur toutes les autres [...] ; il s'agit de la construction d'un ethos pour l'énonciateur-citateur (qu'il soit un habitué des salons mondains, un enseignant universitaire, un homme ou une femme politique, etc.).

8. Visée unifiante ainsi nommée en écho au schème sublinguistique de Gustave Guillaume. [Jaubert, Rosier $2011:$ 10, idt].

38 Selon un mouvement qu'on peut suivre au fil des intertitres, de « Echanges rapportés comme support de l'argumentation [...] » à « Echanges rapportés et construction de l'ethos du locuteur rapportant».

$39 C f$. " proposer un argument, se positionner pour ou contre un autre en faisant jouer la "fonction d'autorité” » (Vincent 2004 : 240) ; « le locuteur utilise les propos d'autrui pour convaincre l'interviewer » (ibid. : 244). 


\subsection{Une composante du dire : le reflet de sa traversée du déjà-dit}

\subsubsection{L'expérience subjective de l'hétérogénéité constitutive du dire}

L'idée de discours isolé, autonome, rejoint dans l'inconsistance l'« Adam mythique » de Bakhtine, prenant la parole dans un monde vierge de tout dire... Un fait de discours n'existe que dans son rapport à d'autres discours : formulée ici de façon triviale, cette loi du discours est celle qui se dégage des deux pensées de l'hétérogénéité constitutive évoquées plus haut (chap. 10) - celle de l'extériorité interne d'un discours déterminé par l'interdiscours et celle de la traversée dialogique du milieu du déjà-dit des autres discours dans lequel prend forme le discours.

S'il n'est pas question d'édulcorer le caractère radical de l'inaccessibilité, pour les sujets, du « tout » des ailleurs discursifs où et d'où se forme leur dire et, spécifiquement, de ceux par lesquels « ils sont parlés » (méconnaissance salutaire sans la protection de laquelle une parole ne peut se tenir, $c f$. chap. 14), il convient de faire droit, pleinement, au versant de l'expérience, subjective, à des degrés divers de conscience, des ailleurs discursifs rencontrés - heurtés, salués, suivis, croisés, frôlés, évités, soupçonnés..., ignorés aussi comme autres - dans l'avancée d'un dire en quête de " ses mots ».

L'éclairage de Bakhtine est ici précieux : contrepartie positive au caractère marginal de la part qu'il concède à l'involontaire, à « l'obscur », dans l'articulation du dire au déjà-dit, c'est clairement le plan de ce qu'on pourrait appeler « le sentiment » ou « l'expérience dialogique » que privilégie Bakhtine ( $c$. ci-dessus chap. 10.1.2, p. 382), revenant inlassablement - et avec bonheur - sur les aventures « vécues », les affects ressentis par une conscience individuelle sur le chemin de son dire à travers le milieu du déjà-dit. Dans l'explicitation de ce point de vue :

La manière individuelle dont l'homme construit son discours est, pour une part considérable, déterminée par la sensation personnelle qu'il a du mot de l'autre et par les moyens qu'il a d'y réagir [Bakhtine 1929/1963, trad. fr. L’Âge d'homme, Lausanne, 1970 : 229].

on note (tout en faisant place à la responsabilité individuelle du traducteur... ${ }^{40}$ ) que c'est jusqu'au niveau corporel de la « sensation » que - à mon sens pertinemment - est envisagée la réaction individuelle au « mot de l'autre » - ouvrant notamment sur les affects irraisonnés, ancrés dans l'intime singularité de l'in-

40 Pour le terme « oščuščenie » susceptible d'être traduit aussi par « sentiment, impression... (communication personnelle de E. Velmezova). 
conscient, source de refus phobiques à énoncer tel mot ou, à l'inverse, de bonheur indicible à user de tel autre.

On observe aussi que cette approche n'interdit pas de prendre en compte - au titre de la « sensation personnelle » qu'un discours « va de soi » - l'insu pour le sujet de la détermination de ses mots par un interdiscours qui, pour une part, «le parle ».

La reconnaissance théorique du fait de l'hétérogénéité constitutive du discours, comme loi, contrainte à se produire dans le milieu du déjà-dit des autres discours et de la détermination du discours par l'interdiscours, n'efface en rien - et ne dispense pas de prêter attention à - la réalité de l'expérience subjective, singulière, que les sujets énonciateurs font de l'avancée de leur dire comme traversée du déjà-dit. De ce déjà-dit qui est celui de sa mémoire discursive spécifiquement sollicitée par les conditions contextuelles de son discours - situation, destinataire, genre, visée du propos, etc. - l'énonciateur va ( $c f$. chap. 14-15), selon sa sensibilité singulière à l'épaisseur sédimentée du langage, en éprouver plus ou moins consciemment la consistance, la résistance, la complaisance.

\subsubsection{Deux filières pour les réactions de l'énonciateur au déjà-dit}

La réaction au déjà-dit qui, dans l'avancée du dire s'impose, se propose, s'oppose... relève de l'une ou l'autre de deux filières par lesquelles passe le dire en train de se faire : celle (a), muette et constante, des réactions « intérieures », conditionnant secrètement le dire, et celle (b) saillante et ponctuelle des réactions extériorisées (de la RDA) qui l'accompagne au plan métalangagier.

(a) Derrière le fonctionnement secret de la première filière de réaction au déjàdit, croisé au fil de l'avancée du dire, se déploie un continuum entre deux pôles :

- l'un est celui de la réaction la plus nettement consciente de l'énonciateur poursuivant son chemin en adoptant, assimilant, sans bruit, le discours autre rencontré, ou au contraire en l'écartant, via une évaluation inexprimée ; évaluation dont on peut surprendre l'expression en dehors du fil même du discours, en avant-texte par exemple, dans des échanges oraux de rédaction à plusieurs explicitant une réaction au déjà-dit («Non, pas ça, ça fait... »), ou, dans les traces que gardent les brouillons, les hésitations et ratures attribuables à un jeu de déjà-dit ( $c f$. ci-dessus, chap. 11.2.3.3, p. 455, pour cause de déjà dit importun, l'exemple d'une rature de Sartre) ;

- l'autre est celui de la non-conscience, c'est-à-dire chez l'énonciateur, d'une « non-réaction » à de l'extériorité discursive, éprouvée non pas comme telle, mais comme discours " allant de soi », normal, inquestionné et propre : la «sensation personnelle » que l'énonciateur a, dans ce cas, « du mot de 
l'autre » est celle d'une évidence familière où se dissout le sentiment de l'extériorité méconnue qui le parle à son insu. Entre les deux, un dégradé serré de niveaux de conscience dans les réactions de l'énonciateur au déjà-dit en jeu dans son propre dire, telles que celles, en retour « désappropriant » sur son dire (déclenchées par l'interlocuteur, ou en après coup...) évoquées chap. 11 ; et, au voisinage du pôle de radicale - et salutaire, redisons-le - méconnaissance, à l'abri de laquelle un énonciateur peut s'engager dans sa parole, celle d'un dire qu'effleure le soupçon, impression fugitive, sentiment indéfini, de n'être qu'un " écho » - à même, de devenir, pour certains énonciateurs, de façon normale ou pathologique (cf. chap. 13), le cœur de leur rapport au langage.

(b) Au traitement de l'extériorité discursive qui s'effectue « en soute » de l'avancée du dire - ou, si l'on veut, dans sa «salle des machines » - répond, à ciel ouvert - sur le pont... - la réaction extériorisée, consciente, dite, de la RDA, offrant, réglée depuis l'étage métalangagier du dire, puissamment sélective, la représentation - au sens aussi de mise en scène - des discours autres élus par le Discours, invités, convoqués... au cours de son cheminement «accompagné ».

Ainsi, du fond du dire prenant corps et se frayant en silence son chemin dans l'épaisseur d'un déjà-dit qui, immaîtrisable par le sujet, pour une part « le parle », se détache la voix de l'énonciateur $\mathrm{L}$, faisant place dans son discours à des discours autres dont il parle.

\subsection{La RDA : une fonction auto-configurante du Discours par ses autres}

\subsubsection{Le Discours « en société » de la RDA}

La RDA fait se lever dans un Discours une société d'autres discours qu'il compose, dispose et met en scène à son gré.

Par rapport à ce qu'il en est de la réalité du dire en dialogue avec d'autres, c'est-à-dire des rencontres - accords, affrontements - imprévues, des surgissements in vivo de l'altérité de la parole d'énonciateurs de chair et d'os, les discours autres mis en place par le Discours sont « ses créatures » qu'il fait surgir par sa parole, sans se départir de la position de surplomb depuis laquelle il sera toujours celui qui les « fait parler » quand il prétend ou s'efforce de leur « laisser la parole » (cf. chap. 4.2.1). À produire, en lui-même, écho métadiscursif de sa traversée de l'extériorité discursive, l'image d'une "société de discours autres », c'est, selon le mécanisme évoqué plus haut - indirect, différentiel, interne - jouant de la variété illimitée des images d'autres mises en place par le Discours, une image de lui- 
même « en société ", c'est-à-dire à travers sa mise en rapport avec ces autres que le Discours fait, secondairement, apparaître.

Aucun discours n'échappe à ce que j’ai appelé la « traversée » de son extériorité discursive, non plus qu'au passage par les deux filières de réaction à cette extériorité qui s'offrent à lui - silencieuse sinon insue vs consciente et dite. Aussi, tout discours présente-t-il - émergence de cette traversée à la surface du dire - son image des autres rencontrés et, partant, l'image de soi qui s'en dégage.

On peut ainsi considérer que tout discours comporte une image de lui-même, configurée par sa mise en rapport (en société) avec ces autres que - parmi tous ceux qui jouent en lui et se pressent aux portes de son dire - il a choisi d'accueillir ; et que la RDA remplit dans le dire - exercée sous couvert de visées discursives diverses (cf. plus haut) - une fonction, partie prenante de l'économie énonciativo-discursive : fonction métalangagière de représentation auto-configurante $d u$ discours (et de son énonciateur) par ses autres.

Dans cette perspective, les discours «monologiques » qui, évidemment sont pétris, comme tout discours, de discours autres - ne sont pas des discours dans lesquels cette fonction cesserait de s'exercer, mais des discours puissamment auto-configurés comme "sans autres », comme discours du UN, du vrai sans partage : que cet « un » soit (dangereusement) de l'ordre - psychique ou idéologique - d'une surdité radicale, inconsciente ou délibérée aux discours autres, ou que, différemment la clôture sur soi du discours relève fonctionnellement de ce que M. Pêcheux appelait les « univers discursifs logiquement stabilisés » :

inscrits dans l'espace des mathématiques et des sciences de la nature, dans celui des technologies industrielles et biomédicales, et dans la sphère sociale des dispositifs de gestion-contrôle administratifs [...] [Pêcheux 1982 : 19]

ou, plus loin encore de la discursivité ordinaire, de l'inscription dans un projet de discours entièrement formalisé, selon un principe - d'altière autosuffisance d'auto-engendrement interne à partir d'axiomes explicites ${ }^{41}$.

41 Tel celui, explicité par N. Bourbaki, au Livre I des Éléments de Mathématique (Hermann, Paris, 1954) : Cf. Authier (1981: 137) :

Mode d'emploi de ce traité : Le traité reprend les mathématiques à leur début et donne des démonstrations complètes. Sa lecture ne suppose donc, en principe, aucune connaissance mathématique particulière. 


\subsubsection{L'image du Discours, reflet des images de ses autres}

Le positionnement du Discours " par ses autres » - « Citer c'est se situer » dit Quéré (1992 : 97) - est, tout entier, jeu d'images et de reflets. L'image du Discours qui se dégage de la "société d'autres » qu'il s'est composée par la RDA est celle - non dite - que reflètent les images de ces autres discours. Dans le Discours, chaque forme de RDA inscrit localement sur la chaîne, dans la matérialité du dire, une image de discours autre : au-delà du qui, de l'identification d'un $\boldsymbol{l}$ distinct de $\mathbf{L}$, chaque image comporte le comment de la façon dont - dans l'immense clavier des formes à travers lesquelles peut se faire l'image et des rapports possibles entre le Discours et tel de ses autres - l'autre discours se trouve, singulièrement, articulé au Discours et par là « l'angle » sous lequel s’y reflètera le Discours.

Le travail de l'image de soi qu'accomplit le Discours par sa RDA repose ainsi - loin du « miroir » de l'ARD offrant directement une image du Discours - sur un jeu spéculaire complexe, " miroir de son être dans l'autre ${ }^{42}$, dans lequel chaque image de discours autre délivre, en retour, une facette de l'image du Discours qui l'énonce ; et où c'est de l'ensemble de ces images de discours autres que le Discours dépose en lui - aussi multiples et disparates soient-elles -, que se dégage, différentielle, composite, indirecte et immatérielle, une image de soi.

Cette différence entre les deux plans, dans un Discours, de la représentation de discours autre qui s'y énonce, localement et sur la chaîne, et de la présentation de soi qui s'en dégage, c'est-à-dire entre ce qui relève du dit et ce qui n'en est qu'un reflet, une émanation, est déjà présente dans la classique formule du « dis-moi qui tu cites... » : la promesse du « je te dirai qui tu es » signifie bien que si l'énonciateur est à même de dire les autres auxquels il fait place, il peut, en revanche, être nécessaire de lui révéler l'image de lui qui se forme dans le jeu de facettes identitaires que sa RDA met en place.

C'est ce qu'indique une formulation comme celle de M.-M. de Gaulmyn $(1996$ : 28) :

[...] le DR « reflète » et « dénonce » la situation de discours et les positions respectives du narrateur et du narrataire.

Et, dans l'analyse aiguë que F. Gai (2011) propose de l'œuvre mauriacienne en tant que « constellée de citations », je retiendrai la distinction qu'il marque - dans le « processus identitaire » qu'il y fait apparaître - entre le plan de la présence matérielle des citations :

42 Cf. ci-dessous 13.1.2.2, p. 496, Dolto au sujet du stade du miroir. 
[...] fréquentes dans le texte, elles donnent corps à l'autre, lui assurant une présence auprès de l'écrivain (Gai 2011 : 38, idt)

et celui de « l’image » que leur jeu « délivre » :

[...] assemblage et collage de discours [...] permettant à l'auteur de délivrer une image de soi (ibid. : 48, idt).

\subsubsection{Au terme d'un parcours : retouches au « Dis-moi qui tu cites, je te dirai qui tu es "}

Dis-moi qui et comment... je te dirai l'image qui se dégage de ton discours

La RDA - dispositif, interne au Discours, de configuration différentielle de soi à partir des discours autres qu'il présente - apparait comme un puissant mécanisme de figuration individuante du Discours et de son énonciateur. Son pouvoir propre - par rapport aux autres ressorts de positionnement - tient, pour une part, à la façon dont s'y articule le dit, interne au Discours, des autres avec lesquels, sélectivement, il se montre en relation, au non-dit de l'image de lui-même qui, déterminée par le jeu de reflets disposé par l'énonciateur, a pourtant l'évidence de ce qui semble s'imposer « tout seul».

Son autre atout majeur est la palette illimitée où puise le Discours, pour représenter ces autres en rapport avec lui, dotant le jeu de reflets où se compose son image d'un pouvoir extrême - dans la précision aiguë, la finesse délicate - de caractérisation différentielle. L'importance du rapport établi dans le Discours avec chacun de ses autres impose une première « retouche » au dicton du « dis-moi qui tu cites... ». Les images de discours autres dont le jeu « dénonce », « délivre », « reflète » l'image du Discours qui les a disposées en lui, ne se réduisent certes pas à l'identification du qui convoqué : le comment de l'accueil qui lui est fait est évidemment essentiel ${ }^{43}$.

On pourrait multiplier les exemples de Discours dont l'image - fort différente - se forme au reflet de l'image d'un même autre, disposé d'une autre façon. Tel, pour prendre un cas saillant, le face à face de textes, également saturés d'un discours autre d'un même « qui ? : Lacan », qu'opposent, radicalement, leurs deux « comment » : celui d'un psittacisme de disciples dévots d'un côté et, de

43 Comme le marque J. Bres (1999 : 84) dans cette formule : « Dis moi quels sont tes autres et comment tu les traites et je te dirai qui tu es... ». 
l'autre, celui d'une mise en pièces par L. Irigaray, dans sa diatribe en forme de « Lettre ouverte à Messieurs les psychanalystes ${ }^{44}$.

La pertinence des différences dans le « comment » de l'accueil fait à l'autre discours ne se réduit évidemment pas à des oppositions axiologiques aussi frontales. C'est tout un ensemble de variables (évoqué ci-dessus, note 29 et chap. 14, 15) qui est en jeu dans chaque fait de RDA, et au-delà dans l'ensemble des faits de RDA dans un Discours, d'où se dégage son image : quels discours autres, en quel nombre, identifiés avec quel degré de précision, inscrits dans quel rapport avec le Discours, à travers quel type de contact (i.e. de mode de RDA), occupant quelle étendue dans le Discours, etc. ; l'exploration des possibilités offertes par chacune de ces variables et, au-delà, par leur combinaison dans un Discours, peut fournir une grille utile à « l'approche des Discours par leur RDA », et y ouvrir des questionnements. C'est sur l'ébauche de ce « programme » que s'achèvera, ci-dessous chap. 15, notre parcours.

Notons que le jeu des variables caractérisant les discours autres et, par elles, l'image du Discours, amène aussi à retoucher le « qui »: les images de discours autres renvoient, aussi bien qu'à des personnes, à des genres de discours, des parlers, des variétés... et, bien sûr, tel propos particulier ; c'est ce que prend en compte l'alternative qui/ce que proposée par Quéré (1992 : 96) :

Dis-moi ce que - ou qui - tu cites, je te dirai qui - ce que - tu es

\section{Dis-moi... et qui tu ne cites pas}

Si l'image qu'un Discours, un énonciateur, donne de lui à travers la RDA constitue assurément un élément de ce qu'il « est », on ne saurait prendre l'une pour l'autre... Il faudrait, entre autres, pour, derrière l'image proposée, approcher (certes pas atteindre) celui - Discours, énonciateur - qui la propose, se demander qui - ce que - il ne cite pas.

L'image donnée, pertinente en elle-même, ne prend tout son sens, on l'a vu chap. 11.1, p. 422, que sur le fond de déjà-dit de la mémoire discursive qui joue en silence dans le Discours, que cette altérité y soit tue délibérément ${ }^{45}$, ou simplement (à des degrés divers et au deux sens du mot : ne pas tenir compte et ne

44 Cf. « Un discours offensif, tout entier sur le mode du comme vous dites » (Authier-Revuz, 1995/2012: 219-224)

45 Par «nécessité performative » [cf. chap. 11 (45), p. 460] pour les formules consacrées, ou par le non-dit calculé du recours à une mémoire soutenant d'autant mieux le projet d'un Discours qu'il demeure inavoué [chap. 11 (30), p. 447]. 
pas savoir) ignorée, comme « allant de soi » - condition même de la possibilité de tenir une parole.

$\mathrm{Si}$ « dis-moi qui tu ne cites pas » serait la plus absurde des requêtes adressée à un énonciateur ne voulant et surtout ne pouvant y répondre, le travail interprétatif de la réception d'un Discours et de sa RDA ne peut ignorer la mémoire discursive qui les habite - que celle-ci soit intuitivement et implicitement prêtée au Discours par le récepteur, à partir de la sienne propre, ou que cette mémoire soit l'objet d'une construction réglée selon le projet et la méthodologie de l'Analyse de Discours (chap. 10.2, p. 393).

Précieuses de ce point de vue sont les approches de la RDA en discours qui s'efforcent de conjoindre le déjà-dit représenté au fond - constitutif - d'où il émerge, ou, si l'on veut, celles qui, dans un discours, prêtent attention au complémentaire ( $c f$. chap. 11) de $\boldsymbol{E}$ ' dans $\boldsymbol{E}$.

Divers travaux permettent de mesurer combien une prise en compte solidaire - plus ou moins systématisée - des deux plans où joue, dans des Discours, leur extériorité, ouvre sur ceux-ci des perspectives, fermées à l'étude d'un seul plan. On a déjà évoqué (chap. 11.1, p. 423) l'éclairage qu'apporte sur le « Journal » de Léautaud, ou sur les discours autoritaires « monologiques », la mise en rapport de « ce qu'ils citent à « ce qu'ils ne citent pas », comme aussi, explicitement inscrite dans cette démarche ${ }^{46}$, l'étude menée par F. Hailon (2011b) d'un corpus de quatre quotidiens pendant la campagne présidentielle de 2002 : le discours du Front National y est envisagé comme une région de l'extériorité discursive dans laquelle se produisent les divers organes de presse ; et c'est par le moyen d'une minutieuse comparaison de ce qui, de ce discours («forces de sécurité », " zones de non droit », « quartiers sensibles »,...) se trouve « cité » ou « pas cité », c'est-à-dire mis à distance comme autre par des guillemets de MAE ou bien pris en charge, « naturalisé », qu'est évalué le degré de pénétration de l'idéologie frontiste dans le paysage médiatique. Sur un autre mode, on observe combien l'analyse d'écrits relevant du « travail social » - rapports éducatifs, signalement d'enfants en danger - gagne en profondeur à être menée aux deux plans de la mise en scène qu'ils offrent par la RDA des propos des divers acteurs concernés - parents, enfants, professionnels divers ${ }^{47}$ - et de l'emprise sur ces écrits de la mémoire interdiscursive hétérogène - où voisinent guides de rédaction, textes juridiques et administratifs, traitements médiatiques de la maltraitance, « fond » culturel, littéraire sur le thème de l'enfance malheureuse, discours «psy » professionnels et de magazines... - dont ils

46 En référence à l'articulation hétérogénéité montrée/constitutive de Authier (1995/2012, $2000, \ldots$...) et à l'approche des domaines de mémoire dans les médias de Moirand (2004).

47 Cf. Sitri (2008), Cislaru et Sitri (2008). 
sont « tissés » et qui s’y manifeste notamment en formulations stéréotypiques et « routines ${ }^{48}$.

\subsubsection{Du qui tu cites... au fait que tu cites : du rapport aux autres au rapport au langage}

On a vu comment, en convoquant tels discours autres en fonction des buts - rhétoriques, narratifs,... - qu'il poursuit, un Discours dispose en lui-même les éléments propices à sa " figuration » différentielle - l'opération d'identification se réalisant parmi et par ces autres, les semblables qu'il s'est choisi comme société et au reflet de l'image desquels se dégage une image de lui-même. Ressort de base de la fonction configurative de la RDA dans le discours, ce mécanisme de construction identitaire, foncièrement spéculaire, appartient au registre subjectif de l'imaginaire, comme lieu du « moi par excellence » et de « la relation duelle à l'image du semblable » dont relèvent les « phénomènes d'illusion, de captation, de leurre, etc. ${ }^{49}$, liés à la construction de cette instance du sujet.

Mais, relevant aussi du registre imaginaire - de l'illusion nécessaire au sujet - auquel s'accorde spécifiquement la position métadiscursive de surplomb de l'énonciateur par rapport au discours, la fonction configurative de la RDA opère encore sur un autre plan : celui, en deçà du rapport à d'autres discours et, se jouant à travers lui, du rapport du sujet énonciateur au langage. L'action du « Faire citationnel » dans le Discours (pour reprendre la formulation d'H. Quéré (1992 :92) n'est pas tout entière comprise dans ces jeux d'images composant, différentiellement, l'image du Discours dans son rapport aux autres discours qu'il représente : en deçà - et au travers - d'un « qui tu cites » menant à « qui tu es », c'est-à-dire de l'image d'un Discours identifié, caractérisé par la représentation qu'il donne de tel rapport, à tel discours, se profile un simple «tu cites »- peu importe qui - faisant apparaître, par contraste, « que tu es » énonciateur d'une parole propre ; « citer » trace dans le Discours une frontière entre ce qui, globalement, quels que soient les autres discours représentés, relève du « dehors » de l'altérité-extériorité discursive et ce qui, délimité par opposition, se trouve par là même institué comme le «dedans » d'une parole " de soi ».

Etudiant le discours rapporté chez P. Modiano, c'est à ces deux niveaux que M. Colas-Blaise (2004) situe ce qu'engage un « style citationnel » - celui où représenter le discours de l'autre permet, par différence, de forger « une identité de

48 Cf. Cislaru et Sitri (2012), Sitri (2015a).

49 Roudinesco et al. (1997), articles Imaginaire (p. 482) et Symbolique (p. 1042) ; cf. ci-dessus chap. 10.3.2, p. 411, 3.4.1, p. 414, et, ci-dessous, 13.1.2.2, p. 496, l'infans au miroir : « moi » aux côtés « d'un autre ». 
discours », et celui, " plus fondamentalement » où se joue, en rapport avec « une conception du langage et de la relation à autrui », " une identité de soi comme sujet énonciateur » (p. 167, 171)...

Avec ce deuxième plan de l'action configurative de la RDA dans le Discours, on quitte donc l'espace de la relation spéculaire à autrui - où un discours s'individualise par rapport à tel(s) autre(s) discours - pour celui du rapport du sujet au langage : celui - contre la menace de dépossession que constitue le réel de l'hétérogénéité constitutive - de l'assurance pour le sujet, aussi illusoire qu'elle lui est nécessaire, de tenir une parole propre.

C'est donc à ce double plan que la fonction configurative de la RDA fait jouer la " positivité du négatif » : les discours autres, intentionnellement convoqués dans un Discours au titre d'éléments de stratégies communicationnelles diverses, y inscrivent, comme corps étranger, « dissimilé », cette part d'altérité (de négativité) qui est condition de la configuration - positive - du discours par lui-même, passant par le travail, d'une double « frontière instituante » :

- la première, envisagée dans ce chapitre, au long de laquelle, par le jeu des images que le Discours propose de chacune de ses rencontres avec des autres spécifiques, se forme l'image à multiples facettes par laquelle le Discours se différencie, se caractérise, se positionne, s'identifie comme un parmi et par rapport à ces autres ;

- la seconde, issue de la première, qui délimitant dans le Discours la part attribuée à la représentation de discours autre, institue par là l'espace de son complémentaire comme celui - imaginaire - $d u U N$ sans autre d'une parole propre, assurant ainsi au sujet « l'assiette » subjective d'une parole énoncée - depuis le dit ailleurs-avant-et indépendamment dont, pourtant, elle est faite - comme «sienne », porteuse de la contradiction inhérente au langage - qu'on tentera d'approcher dans la suite.

Ainsi le mécanisme métalangagier de représentation de discours autres dans le Discours, traverse-t-il plusieurs plans, distincts et articulés, passant de celui, intentionnel, des stratégies communicatives (évoqué en 2.1), à celui, très inégalement conscient, du travail de l'image de soi parmi ses autres (évoqué en 2.3), pour déboucher dans celui où se joue, insu, quelque chose du rapport du sujet au langage (interrogé dans ce qui suit). 